\title{
MATILDA O LA HISTORIA DEL LOBO BAJO LA PIEL DEL CORDERO
}

\author{
María Aída Díaz BiLd
}

Universidad de La Laguna

\section{RESUMEN}

En los últimos años la crítica feminista ha reivindicado la importancia de la obra de Elizabeth Inchbald y más concretamente de su producción novelística, que incluye A Simple Story (1791) y Nature and Art (1795). De estas dos novelas la que más interés ha despertado es $A$ Simple Story y en especial el contraste entre sus dos heroinas, Miss Milner y Matilda. La mayor parte de la crítica coincide en que, frente a Miss Milner, cuya actitud es abiertamente subversiva, Matilda representa el ideal de sumisión y obediencia imperante en el siglo XVIII. Yo creo, sin embargo, que el comportamiento de Lady Matilda y su amado, Rushbrook, guarda importantes concomitancias con el de la heroína y el héroe del género gótico y que es precisamente este aspecto el que hace que la segunda parte de $\mathrm{A}$ Simple Story no sea una historia tan conservadora como se nos ha hecho creer, sino un relato iconoclasta que a través de una fantasía de poder intenta mostrarle al lector cómo deberían ser las cosas en una sociedad más justa.

Elizabeth Inchbald, actriz, dramaturga, novelista, traductora y clara simpatizante de los denominados autores jacobinos, gozó de gran prestigio y reconocimiento como escritora en la última década del siglo XVIII. Ya en 1810 An- 
na Laetitia Barbauld, una de las más fervientes defensoras no sólo de la novela como género literario, sino de la decisiva aportación de la mujer al mismo, y la editora de British Novelists, with an Essay and Prefaces, Biographical and Critical, destaca el papel fundamental que Inchbald ha desempeñado en el desarrollo de la novela:

And indeed... it may safely be affirmed that we have more good writers in this walk living at the present time, than at any period since the days of Richardson and Fielding. A very great proportion of these are ladies: and surely it will not be said that either taste or morals have been losers by their taking the pen in hand. The names of D'Arblay, Edgeworth, Inchbald, Radcliffe, and a number more, will vindicate the assertion. (cit. en Moore 1986: 388).

Barbauld alaba la originalidad de Inchbald tanto en lo que a la creación de personajes como de situaciones se refiere así como su maestría en la manipulación del tiempo y del punto de vista. También María Edgeworth, una de las escritoras más aplaudidas del siglo de las luces, expresa de forma un tanto apasionada su admiración por los logros artísticos de Inchbald. Edgeworth reconoce que ha leído cuatro veces $A$ Simple Story, la novela más conocida de Inchbald, definiéndola como «the most pathetic and the most powerfully interesting tale I ever read» (cit. en Figes 1988: 169), y ensalza la capacidad de Inchbald para crear personajes verosímiles.

Otro personaje ilustre de la epoca que defiende las excelencias de Inchbald como escritora es Erasmus Darwin. En su A Plan for the Conduct of Female Education at Boarding Schools (1798) clasifica las novelas en «serious, humorous, or amorous» (cit. en Armstrong 106) y decide incluir las de Inchbald dentro de la primera categoría, que para él es la más excelsa de las tres.

En los últimos años la crítica feminista, fiel a su empeño por recuperar los textos de las autoras del siglo XVIII, cuya voz había quedado silenciada con el paso del tiempo, ha reivindicado la importancia de la obra de Inchbald y más concretamente de su producción novelística, que incluye A Simple Story (1791) y Nature and Art (1795). De estas dos novelas la que más interés ha despertado es A Simple Story, que Casle no ha dudado en definir como «a tour de force - a small masterpiece neglected for too long. Without exaggeration the case might be made for $A$ Simple Story as the most elegant English fiction of the century» (290). La primera parte de la novela se centra en Miss Milner, una muchacha frívola que se enamora de su tutor, un sacerdote jesuita, enfrentándose así a las leyes de la Iglesia católica y violando algo tan sagrado como es la relación entre tutor y pupila. Cuando todos los impedimentos para su unión 
desaparecen al tener que renunciar Dorriforth a sus votos para poder heredar un título nobiliario y convertirse en el nuevo Lord Elmwood, Miss Milner, no contenta con tener el cariño y la pasión de su amado, decide poner a prueba la paciencia de éste y desafiar su autoridad, yendo a un baile de máscaras, al que Lord Elmwood le ha prohibido asistir. La heroína desobedece la orden de su futuro marido y ello provoca la ruptura de la pareja. Sin embargo, de una forma un tanto «milagrosa» esta primera parte termina con la unión de los dos enamorados.

Cuando la segunda parte comienza han transcurrido diecisiete años y Miss Milner se encuentra en su lecho de muerte. Hace muchos años que vive con su hija Matilda en un lugar inhóspito de Escocia, repudiada por su marido por haber cometido adulterio al poco tiempo de estar casados. Miss Milner muere y Lord Elmwood permite que Matilda se instale en su casa bajo la condición de que no se produzca nunca un encuentro entre padre e hija. Si tal situación se diera, Matilda tendría que abandonar inmediatamente el hogar paterno. Matilda acata con total sumisión la orden de su padre, pero, lamentablemente, un día tropieza de forma accidental con Lord Elmwood y es expulsada de la casa. Margrave, que representa al clásico villano de las novelas sentimentales, se aprovecha del estado de indefensión en que se encuentra la muchacha, y la rapta. Ello despierta el amor paternal de Lord Elmwood que acude en ayuda de su hija y la novela termina felizmente con la reconciliación de padre e hija y la unión de ésta con su incondicional amado, Rushbrook.

La actitud desafiante de Miss Milner contrasta con la conducta sumisa de Matilda y ello ha llevado a la crítica a resaltar el carácter subversivo de la primera parte frente al talante conservador de la segunda, con una sola excepción, Terry Castle. Frente a autores como Kelly, que considera que Matilda refleja una superioridad moral de la que carece su madre al aceptar con sumisión su situación, o Tompkins que afirma que Miss Milner es un personaje totalmente vital frente a Matilda cuya historia es inferior a la de su madre, Castle defiende no sólo que Matilda es la perfecta sustituta de Miss Milner, sino que la estructura narrativa de la segunda parte es casi idéntica a la de la primera: «Matilda's story not only resembles her mother's, it is a displaced recapitulation» (323). Según Castle, el mismo patrón de prohibición/violación/recompensa se da tanto en el caso de Miss Milner como en el de Matilda. Mientras que a la primera Lord Elmwood le prohíbe ir al baile de máscaras, a la segunda no le permite transitar por determinadas habitaciones de la casa cuando él está. Y al igual que Miss Milner viola las reglas asistiendo a la fiesta de disfraces, Matilda transgrede las normas yendo a la biblioteca de la casa en su ausencia, mirando sus retratos, escuchando el ruido de su carruaje $y$, finalmente, encontrándose con él, aunque sea de forma accidental. Matilda, como su madre, ha 
de pagar la falta cometida, pero tras abandonar la casa paterna Inchbald busca la manera para que se produzca la reconciliación entre padre e hija. Para Castle la principal diferencia entre la primera y segunda parte de la novela es que en ésta última la zona prohibida no es ya algo externo:

Matilda's story represents not a disavowal but an internalization of the carnivalesque. The transformational energy of the masquerade in the second half moves into the private world of the bourgeois household, and on a subjetive level, into the realm of individual psychology (325-6).

Ty coincide con Castle en resaltar el carácter subversivo de $A$ Simple Story, pero es menos optimista con respecto al final de la misma. Partiendo de la teoría psicolinguística de Lacan y las tesis feministas psicosociales de Chodorow, Ty afirma que la historia de Miss Milner es ante todo la de una joven que se niega a formar parte del orden simbólico, mientras que su hija está dispuesta a aceptar la ley del padre y adoptar, por tanto, una postura de sumisión y docilidad. Al igua que Castle, Ty no cree que Inchbald esté presentando a Matilda como el ejemplo a seguir, en contraste con su madre, que parece haber carecido de la educación adecuada, pero sí discrepa con Castle en su visión de Matilda como emblema de liberación. En la segunda parte de la novela entramos en un mundo totalmente diferente en el que la autoridad y el poder masculino no encuentra oposición alguna. A través de Matilda y de su actitud sumisa, Inchbald señala las restricciones que la sociedad impone a las mujeres que desean ser aceptadas en el mundo del padre y describe lo arbitrario, opresivo y amenazante que puede llegar a ser el poder paternal '. En este sentido, para Ty lo que encontramos al final de $A$ Simple Story no es un mundo utópico de libertad, sino una aceptación resignada de la situación de la mujer en el siglo XVIII: «In this way the novel is both a celebration of and an elegy on the energetic possibilities of woman» (100).

Ty es de las pocas estudiosas que dedican también un espacio a Rushbrook, ya que considera que es significativo que Matilda despierte los sentimientos de un joven tan sumiso e indefenso como ella. Rushbrook, sobrino de Elmwood, pedende económicamente de su tío y ello influye de forma decisiva en la relación de ambos personajes: «As a dependent of the figure of authority he is circumscribed by his obligations, gratitude, and loyalty to Dorriforth. His inclination to rebel is checked, and opposition remains indirect» (99). Al contra-

' En términos semejantes se expresa JANET TOOD en The Sign of Angellica cuando afirma: "With this repetition she made - but only by implication - a biting attack on authoritarian patriarchy». (228). 
rio que Miss Milner, Ruhbrook no se enfrenta abiertamente al poder patriarcal y es recompensado por su paciencia y docilidad con la mano de su amada. Matilda y Rushbrook han interiorizado la ley del padre, mostrando así un mundo más represivo y moderado que el que encontramos en la primera parte.

$\mathrm{Al}$ igual que Ty, Spacks rechaza la interpretación gloriosa y liberadora que Castle hace de $A$ Simple Story y, por tanto, no acepta que la segunda parte sea una recapitulación de la primera. Spacks considera que con el comienzo de la historia de Matilda se produce una reafirmación del orden social tradicional. Miss Milner, tras cometer adulterio, acepta el poder absoluto de su marido y Matilda a su vez asume la autoridad de su padre sobre ella: «Taking a rather less rosy view than Castle's, I find in Matilda's career not a narrative of female freedom and power but one of necessary acceptance and limited reconciliation" (199). A diferencia de su madre, Matilda no es un ser pasional que represente una amenaza para el orden social o la sensibilidad de los lectores, y su conformidad con las convenciones establecidas es «justamente» recompensada. Frente a la primera parte que celebra la feminidad pasional, energética, individual, la segunda parece confirmar la necesidad de una autoridad patriarcal dominante. En este sentido, Spacks coincide con Ty al afirmar que en A Simple Story no encontramos falsos triunfalismos, sino la aceptación de la realidad tal y como es: «Her plot embodies the struggle, between anger at the necessities of female compliance and awareness that no viable alternative exists" (202).

Al contrario que Castle y al igual que Ty y Spacks, Spencer considera que la segunda parte es muy distinta a la primera, ya que en ella se nos presenta un ejemplo extremo de tiranía paternal que recuerda claramente la situación dramática de muchas novelas góticas ${ }^{2}$. Matilda no desafía la autoridad paternal o reafirma sus deseos. Para Spencer esta segunda parte era necesaria si Inchbald quería darle un final feliz a su historia, ya que las convenciones literarias del momento no permitían recompensar a un personaje como Miss Milner que con su ingenio y sexualidad había amenazado la autoridad de su tutor.

Al igual que Ty, Spacks y Spencer, Rogers considera que hay evidentes discrepancias entre la primera y la segunda parte de A Simple Story, pero es mucho más crítica con ésta última. Según Rogers, esta diferencia se debe a las presiones bajo las que escriben las mujeres en el siglo XVIII. Si la mujer escritora quería alcanzar fama y prestigio su conducta y sus escritos debían reafirmar los valores y actitudes aceptadas. La mejor manera de mostrar su acatamiento a las normas imperantes era creando una heroína casta, sumisa,

2 TY y SPACKS también han establecido claros paralelismos entre la novela gótica y la actitud represiva de LORD ELMwOOD en la segunda parte. 
delicada, sensible, con un gran sentido del decoro y del deber. Evidentemente, Inchbald ignora esta convención en la primera parte de $A$ Simple Story, al presentamos a una joven protagonista que no se adapta al ideal femenino de la época y que insiste en hacer su voluntad, pero en la segunda parte se siente obligada a adaptarse a los cánones establecidos creando una heroína pasiva y sumisa. De ahí que para Rogers la historia de Matilda carezca de la originalidad y convicción de la de Miss Milner.

Schofield por su parte pone el énfasis en el realismo con que Inchbald describe la situación de la mujer en el siglo XVIII y, más concretamente, en el deseo de la escritora de hacer que sus lectores despierten de su letargo e inocencia y se den cuenta de que están rodeadas de hombres que las manipulan y oprimen. En términos de la ideología masculina, Miss Milner es un ejemplo de lo que no se debe ser, ya que no sólo se niega a que nadie la controle, sino que además intenta poner a prueba su poder sobre su futuro marido. Matilda, por el contrario, acepta ponerse la máscara de la heroína de los romances y acepta voluntariamente el papel de sumisión. De hecho, no sólo su secuestro a manos de Lord Margrave es totalmente predecible, sino que durante su cautiverio Matilda se comporta con relativa calma, como si supiera que está interpretando el papel que la sociedad atribuye a sus heroínas. En este sentido, lo que encontramos en esta segunda parte de $A$ Simple Story es una alegorización de la primera: «Part One tries to present the feminine text, while Part Two is the man's story» (180). Inchbald satiriza el romance romántico y sentimental de mediados del siglo XVIII, poniendo al descubierto los principios en los que se basa este género, principios que sólo llevan a la indefensión e infelicidad de la mujer.

Es indudable que, como afirma la mayoría de la crítica aquí citada, el comportamiento de Lady Matilda se adapta al ideal de sumisión y obediencia imperante en el siglo XVIII y que, en este sentido, puede afirmarse que la segunda parte de A Simple Story es más conservadora y realista que la primera al ofrecernos un retrato más fidedigno de la situación de dependencia de la mujer en esa época. Pero junto a este elemento naturalista se introduce otro de índole claramente utópico o fantástico al ofrecérsele al lector en las últimas páginas de la novela una relación de pareja basada en principios igualitarios y feministas que nada tiene que ver con el tipo de matrimonio imperante en el siglo XVIII, en el que todo el poder residía en el hombre. Es precisamente esta combinación de lo real y lo ideal lo que vincula la segunda parte de A Simple Story con la ficción escrita por mujeres a finales del siglo XVIII y, más concretamente, con la novela gótica. Es cierto, como veíamos antes, que la crítica ha discernido en Lord Elmwood rasgos del tirano de la novela gótica que hace uso del poder que la sociedad le otorga para oprimir a la heroína, pero no ha ido más alla. Yo creo, sin embargo, que el comportamiento de Lady Matilda y su amado, Rushbrook, 
guarda importantes concomitancias con el de la heroína y el héroe del género gótico y que es precisamente este aspecto el que hace que la segunda parte de $A$ Simple Story no sea una historia tan conservadora como se nos ha hecho creer, sino un relato iconoclasta que a través de una fantasía de poder intenta mostrarle al lector cómo deberían ser las cosas en una sociedad más justa.

Uno de los grandes logros de la crítica feminista en los últimos años ha sido la de demostrar que la novela gótica no es un género meramente escapista, sino un vehículo de afirmación femenina. La novela gótica se convierte en manos de la mujer escritora en un género liberador y subversivo que le permite sacar a la luz de forma encubierta sus problemas y sufrimientos:

\begin{abstract}
Alienated as women were from a culture which was male, a culture which denied them identity and isolated them from male society and from each other, women felt weirdly at home in the «other» world of the Gothic novel. And they used the Gothic mode to reveal the terror, the isolation and the oppression of their lives. The Gothic «the discourse of the Other». (Van Leeuwen 1982; 37).
\end{abstract}

Desde que Ellen Moers en su ya clásico Literary Women acuñara el término «female gothic», la crítica feminista se ha interesado por descubrir «what's female about gothic» (Becker 1999: 9) y, particularmente, por la clase de héroe y heroína que este tipo de textos crea. Autoras como Rogers o Figes, entre otras, han afirmado que una de las características de la novela gótica es la creación de un tipo de heroína que, aunque parezca adaptarse a las convenciones culturales y económicas que subordinan a la mujer, presentándola como un ser generoso, asexuado, sensible, etc., sin embargo, a través de su inteligencia, energía y fortaleza subvierte la ideología femenina establecida y los moldes patriarcales, derrotando a los más poderosos. En realidad esta nueva heroína está presente no sólo en la novela gótica, sino en muchas de las novelas que se publican sobre todo en las últimas décadas del siglo XVIII, en las que aparecen personajes femeninos, que a pesar de su indefensión y de ser víctimas de la crueldad de los hombres, logran defenderse de sus acosadores y demostrar su poder.

Pero, evidentemente, una nueva heroína necesita un nuevo héroe, «a refined feminised man, well born but not excessively privileged in his social position. He expresses the sentimental fantasy that unaggressive feminine sentiment can be extended to men.» (Tood 1989: 264). En efecto, el héroe de la novela gótica proviene de la novela sentimental que ha intentado crear un nuevo tipo de hombre que refleje la ternura, sensibilidad y amabilidad que normalmente se asocian con la mujer. Figes ha explicado el porqué de este cambio con gran claridad: 
If women were being educated to become companions to their husbands, it followed that men also had to change somewhat, to become fit companions for their wives. Tenderness, kindness and sensibility were increasingly regarded as male virtues, and the Man of Sentiment made his appearance in fiction. (12-3).

Se huye así del estereotipo agresivo y dominante que prevalece en las novelas y en los manuales de conducta de la época y se crea un héroe convertido a unos principios más igualitarios y feministas.

Una de las autoras que con mayor profundidad ha estudiado la creación de un héroe y heroína diferentes en la novela gótica es Diane Long Hoeveler. Hoeveler coincide con otros críticos en que el género gótico contribuye a la femnización el héroe y ofrece una nueva concepción de la heroína, pero lo hace desde una perspectiva diferente. Hoeveler se desmarca de las lecturas psicológicas o sexuales de las novelas góticas escritas por mujeres e insiste en que todas ellas se definen por una serie de estrategias estéticas y políticas, cuyo último fin es recrear los horrores de la vida patriarcal inglesa de una forma segura y nada controvertida al situar los escenarios en lugares remotos. La tesis principal de Hoeveler es que lo que hoy se denomina «victim feminism», es decir, la noción antifeminista de que la mujer adquiere superioridad social y moral al presentarse como víctima de un tirano corrupto y una sociedad patriarcal, tiene su origen en la novela gótica femenina:

«Victim feminism», like gothic feminism, is a literary ideology and cannot be understood apart from reading its rhetoric within the originating sources - the gothic novels of Charlotte Smith, Ann Radcliffe, Jane Austen, Charlotte Dacre Byrne («Rosa Matilda»), Mary Shelley, and the Brontës. (xiv-xv).

Todas estas autoras contribuyen a la creación de un nuevo rol para la mujer que las ayude a adaptarse al nuevo orden burgués. Con otras palabras, la novela gótica enseña a las mujeres un tipo diferente de conducta, lo que Hoeveler denomina «professional feminity», y que consiste en «a cultivated pose, a masquerade of docility, passivity, wise passiveness, and tightly controlled emotions» (XV). Las escritoras construyen, pues, una serie de ideologías - un grupo de máscaras y poses literarias - que permiten a los personajes femeninos, y también a las lectoras, alcanzar un dominio ficticio sobre el sistema social y político que las reprime. Hoeveler subraya en este sentido que la novela gótica femenina parte del convencimiento de que las mujeres se ven constreñidas no sólo por una política de género, sino también por los espacios sociales, 
económicos, políticos, religiosos y jerárquicos que el capitalismo burgués y, por extensión, por todo aquello que la familia patriarcal ha construido para ellas.

Hoeveler se centra especialmente en el tipo de heroína que crea la novela gótica y sugiere que sus autoras aparentemente adaptan el modelo de feminidad ejemplar, pero de forma soterrada hacen que sus heroínas consigan dominar el mundo que las rodea. De esta manera mantienen la ilusión de que las heroínas se adaptan a los ideales de sencillez, debilidad y servicialidad de la época:

Or, to be more precise, the female gothic novel represented women who ostensibly appear to be conforming to their acceptable roles within the patriarchy but who actually subvert the father's power at every possible occasion and then retreat to studied postures of conformity whenever they risk exposure to public censure. I have come to recognize and label this ideology as «gothic feminism», ... (6)

Una y otra vez las novelistas defienden que sólo una forma codificada de conducta o una «masquerade», es decir, el "professional feminism», podrá ayudar a la mujer a sobrevivir en un mundo que le es hostil. En los textos góticos, pues, se inventa una peculiar forma de feminismo a través de la creación de lo que Hoeveler denomina "a rival female-created fantasy» (7), que le permite rechazar su subordinación y posición como «the other» y, con ello, la ideología masculina sobre la mujer.

Al presentar a la heroína como una víctima inocente y asediada se la protege de cualquier acusación de que ella es realmente la agresora: «Playing the victim often simply conceals the fact that one is a much more effective victimizer» (14). Las mujeres que pueblan las novelas góticas triunfan al final, tanto moral como económicamente, pero en su camino hacia la victoria han causado verdaderos estragos. Sin embargo, nadie las condena, porque han sabido ganarse la simpatía del lector desempeñando perfectamente el papel de víctima inocente:

The gothic feminist always manages to dispose of her enemies without dirtying her dainty little hands. The position that Radcliffe and her followers advocated throughout the female gothic was one of «wise passiveness" or what we might more accurately recognize as a form of passive-aggression. (7)

Las escritoras de novela gótica responden a los cambios económicos, sociales y religiosos que se producen con la llegada de la economía capitalista, creando una fantasía según la cual los humildes adquieren poder, precisamente cultivando esa apariencia de indefensión. Como dice Hoeveler con gran ironia: «The meek shall inherit the gothic earth» (19). 
Antes decía que las heroínas triunfan moral y económicamente al recobrar al final su fortuna y demostrar que aquéllos que las acosaban estaban equivocados. Pero lo que hace que su victoria sobre la sociedad patriarcal sea perfecta es la creación de un "feminized man", que promete ser completamente dócil. Este factor es más importante de lo que se pueda pensar, puesto que para la mujer de clase media un hogar asentado sobre principios patriarcales es una prisión o un manicomio, en el que se la reduce a la mera condición de objeto decorativo o funcional. Pero el hogar que las heroínas crean al final de las novelas góticas es bien distinto, no sólo porque ellas son las que desempeñan el papel dominante, sino porque sus maridos han sido «feminizados». En este sentido, Hoeveler resalta que las heridas que sufre el héroe gótico en la mayoría de las novelas son importantes, porque le ayudan a enfrentarse y rechazar un mundo patriarcal que oprime a la mujer:

But gothic heroes all endure very real beatings and wounds, not merely symbolic ones, and in the receiving of these wounds it is as if they have earned the right to overthrow their fathers and establish a new companionate femily and a redeemed class - a bourgeoisie that has learned to tame its excesses and perfectly balance reason and the emotions. (20)

Hoeveler resalta que escritoras como Smith, Radcliffe o Austen comprenden rápidamente que las heroínas no pueden triunfar sobre las fuerzas corruptas del viejo régimen sin la ayuda de un aliado, el «feminized man»: «They needed male allies; they needed a protection system behind them; they needed feminized husbands». (50). El gran reto es encontrar a ese hombre feminizado, ese marido que pueda ser controlado y en el que sea posible confiar.

Esta concepción de una relación más igualitaria entre el héroe y la heroína está también relacionada con el cambio que, al menos en teoría, se produce en el siglo XVIII del matrimonio concertado al «companionate marriage», basado en la amistad en el siglo XVIII del matrimonio concertado al «companionate marriage", basado en la amistad, el afecto, el respeto y no en el poder absoluto del marido sobre la mujer ${ }^{3}$. La evolución de un tipo de matrimonio a otro no estuvo exenta de polémica, ni se produjo de forma sistemática, de manera que a la vez que unos defendían que la relación de poder entre padres e hijas debía ser modificada, dándole una mayor capacidad de decisión a los futuros contrayentes, otros parecían ignorar los nuevos aires de cambio y seguían reproduciendo en sus historias y novelas el conflicto generacional entre padres e hijas.

${ }^{3}$ En The Family. Sex and Marriage in England 1500-1800 LAWREnCE STONE explica esta evolución de un tipo de matrimonio a otro con gran claridad. 
Las mujeres novelistas no dudan en ningún momento en reflejar este nuevo ideal de matrimonio basado en el amor y el respeto y en una relación de igualdad más que de subordinación. Pero la senda hacia la felicidad no es fácil y las novelas ilustran las dificultades que las protagonistas femeninas encuentran en su camino hacia esa panacea del «companionate marriage». Se rechaza el matrimonio de conveniencia y se insiste en que toda unión debe estar fundamentada en la cooperación dentro de la pareja. La heroína ha de elegir a su marido por criterios puramente personales, y no por razones económicas, familiares, etc.

Es obvio que Inchbald, al igual que otras autoras de la época, era consciente de que este nuevo héroe amable, cariñoso, conocedor de la superioridad de su amada, era una quimera difícil de encontrar en la vida real. Jane Austen en una carta a su sobrina Fanny, que acaba de rechazar a un pretendiente, expresa esta idea con gran claridad:

There are such beings in the World perharps, one in a Thousand, as the Creature You and I should think perfection, Where Grace \& Spirit are united to Worth, where the Manners are equal to the Heart \& Understanding, but such a person may not come in your way, or if he does, he may not be the eldest son of a Man of Fortune, the Brother of your particular friend, \& belonging to your own County. (cit. en Brownstein 1994: 139).

También sabía que, a pesar de que el concepto de una unión basada en el compañerismo y la amistad iba ganando terreno a medida que el siglo iba avanzando, lo cierto es que las leyes y la estructura social seguían siendo las mismas y, por tanto, a nivel oficial la relación entre el hombre y la mujer seguía siendo de índole jerárquica y no igualitaria. Como apuntaba William Blackstone en 1765 en sus Comentaries on the Laws of England:

The husband and wife are one person in law; that is, the very being or legal existence of the women is suspended during the marriage, or at least is incorporated and consolidated into that of the husband: under whose protection and cover, she performs everything. (cit. en Hoeveler 1998: 6).

Lo que las escritoras como Inchbald estaban buscando era una alternativa a ese régimen patriarcal que oprimía a la mujer y puesto que la única opción aceptable que la mujer tenía en el siglo XVIII era el matrimonio ${ }^{4}$, decidieron

${ }^{4}$ La vida de la mujer soltera estaba jalonada de sinsabores y no gozaba del beneplácito social. Además, la mujer carecía de una educación que le abriera las puertas a una vida mejor. 
crear un nuevo tipo de unión conyugal y un héroe diferente. Quizás, como ha dicho Judith Lowder Newton refiriéndose a Jane Austen, se trata simplemente de una fantasía de poder, pero detrás de toda fantasía hay una clara insatisfacción con la realidad y una necesidad de cambiarla y subvertirla.

Matilda es un personaje que parece encarnar a la perfección el ideal de sumisión y humildad de la época. Frente a la frivolidad de su madre, Matilda lleva una vida más austera, en la que no hay sitio para aquellos divertimentos que puedan poner en peligro su honor y reputación y que tan criticados eran en lo manuales de conducta de la época:

Educated in the school of adversity, and inured to retirement from her infancy, she had acquired a taste for all those amusements which a recluse life affords - She was fond of walking and riding - was accomplished in the arts of music and drawing, by the most careful instructions of her mother - and as a scholar she excelled most of her sex, from the great pains Sandford had taken with that part of her education, and the great abilities he possessed for the task. (221)

A pesar de que su padre no ha querido saber nada de ella ni de su madre desde el momento en que ésta cometió adulterio, Matilda, como procede en toda buena hija, admira profundamente a su padre e incluso ve su aprecio hacia él aumentado cuando consiente en admitirla en su casa a condición de que nunca se produzca un encuentro entre ellos: «Even her mother has not a more exalted and transcendent idea of Lord Elmwood's worth, than his daughter had formed; and this little bounty just obtained, had not been greater in her mother's estimation, than it was now in her's.» (218).

Matilda en ningún momento siente rencor porque su padre haya elegido a otro, a Rushbrook, como su heredero y acata las órdenes de su padre sin protestar. Matilda demuestra en todo momento el respeto y amor que se espera de una hija hacia un padre, aunque la conducta de éste no sea digna de alabanza: coge la mano de Miss Woodley que Lord Elmwood ha estrechado y la besa "with love and reverence» (228); no se cansa de mirar el retrato de su padre; toca con auténtica devoción los objetos que éste ha utilizado:

In the breakfast and dining rooms she leaned over those seats with a kind of filial piety, on which she was told he had been accustomed to sit. And in the library she took up with filial delight, the pen with he had been writing; and looked with the most curious attention into those books that were laid upon his reading desk. - But a hat, lying on one of the tables, gave her a sensation beyond any other she experienced on this occasion - in that trifling article of his dress, she 
thought she saw himself, and held it in her hand with pious reverence. (246)

Quizás la actitud de Matilda hacia su padre pueda parecer hoy en día exagerada, pero lo que se esperaba de una hija en el siglo XVIII, tal y como preconizaban los manuales de conducta de la época, era que fuera totalmente sumisa y obediente con sus padres en todos los aspectos de la vida. De hecho, Matilda en lugar de pedirle a Dios que le cambie su situación, le suplica que le de paciencia y fortaleza para soportar el alejamiento de su padre. Esta conciencia de que como hija ha de ser obediente explica por qué cuando accidentalmente se encuentra con su padre y ha de abandonar la casa, lo hace con total sumisión y sin reproches: Matilda sabe que ha actuado mal al desobedecer la orden de su padre de mantenerse fuera de su vista. Ello no quiere decir, por supuesto, que Matilda no sufra al verse obligada a trasladarse a una casa lejos de la de su padre por la infracción cometida: su salud se va deteriorando, cada vez está más delgada y pálida y su expresión es de absoluta melancolía. Pero aún así, sigue sin rebelarse contra la actitud autoritaria de su padre, que está haciendo que su hija pague por algo que ocurrió en el pasado y de lo que ella no ha tenido culpa alguna. De hecho, cuando es raptada por Lord Margrave y Lord Elmwood acude en su ayuda, «She could only turn to him with a look of love and duty; her tongue could not utter a sentence» (329), a pesar de que ha sido la indefensión en la que su padre la ha dejado la que ha propiciado que un villano como Margrave haya intentado aprovecharse de ella.

Pero tras esta fechada de sumisión se esconde una mujer capaz de pensar y actuar por sí misma que en momentos tan dramáticos como el de su rapto demuestra un gran coraje. En este sentido, resulta curioso observar cómo a lo largo de la novela se resalta que Matilda se parece a su padre tanto físicamente como en su manera de ser y algunos de los aspectos que comparten, como son la arrogancia y la soberbia, nada tienen que ver con la humildad y la obediencia. Matilda demuestra la capacidad intelectual y la sensibilidad de la heroína gótica en su trato con Rushbrook. Aunque le envidia y en ocasiones incluso le odia porque recibe todo el cariño y la atención de Lord Elmwood, mientras que ella ni siquiera puede verlo, es consciente de que si su padre le ha nombrado heredero no es a causa de las maquinaciones del joven, sino por lo ocurrido en el pasado con Miss Milner. De ahí que, a pesar de su rencor hacia él, no dude en defenderlo de las críticas de Miss Woodly y Sandford, que le hacen culpable de la desolada situación de Matilda: «Matilda had the understanding to imagine, she was, perhaps, the object who had thus deformed Mr. Rushbrook, and frequently (though he was a stranger to her, and one who had caused her many a jealous heartache) frequently she would speak in his vindication.» (231). Es- 
ta cita demuestra claramente cómo Matilda no es un ser tan pasivo como la crítica nos ha querido hacer ver, sino que es capaz de sacar sus propias conclusiones e incluso actuar por propia iniciativa, aun cuando los que la rodean no aprueben su conducta. Un ejemplo de ello lo tenemos cuando Matilda se encuentra con Rushbrook después de que éste ha estado gravemente enfermo y se detiene para preguntarle cómo se encuentra. Mr. Sandford censura su comportamiento ya que debería haber sido el joven el que saludara primero a la muchacha, pero Matilda con gran decisión le responde: «"Then while he looks so pale", replied Matilda, "and so dejected, I can never forbear speaking to him when we meet, whatever he may think of it."» (266).

Pero el momento de la novela en que Matilda con más fuerza revela su coraje y capacidad para defenderse, demostrando así que no es un ser tan pasivo, es cuando Margrave la rapta y ella se enfrenta abiertamente a él:

The extreme disgust and horror his presence, inspired, caused Matilda for a moment to forget all her weakness, her want of health, her want of power; and rising from the place where she sat, she cried, with her voice elevated, «Leave me, my lord, or I'll die in spite of all your care; I'll instantly expire with grief, if you do not leave me». (327)

Este episodio resulta interesante no sólo porque tras esa fachada de sencillez y servilismo Matilda revela una gran valentía y una superioridad intelectual y moral poco común, sino porque, como explica Hoeveler, en su camino hacia el triunfo, es decir, hacia la reconciliación con su padre y la recuperación de la herencia al casarse con Russhbrook, la heroína se va deshaciendo de aquellos que han intentado destruirla. Gracias a la intervención de Lord Elmwood, que «súbitamente» ha recuperado su instinto paternal, Margrave tendrá que pagar muy caro por lo que ha hecho. Evidentemente, Matilda no es responsable directa de lo sucedido, ni mancha sus delicadas manos con sangre ajena, pero si no fuera por ella, un ser tan despreciable como Margrave, que al igual que otros hombres de la época, aprovecha la miseria a la que se ven abocadas muchas mujeres al carecer de recursos económicos para robarles su tesoro más preciado, el honor, seguiría actuando con total impunidad.

La infancia de Rushbrook no es en sus inicios más idílica que la de Matilda. Al quedarse huérfano a muy temprana edad, Lord Elmwood decide hacerse cargo de él con la condición de no verlo nunca, ya que la madre del muchacho y hermana de Lord Elmwood se ha casado contra la voluntad de éste último. Pero cuando Miss Milner entra en su vida todo cambia y gracias a su intercesión Lord Elmwood admite al muchacho en su casa. Tras el adulterio de Miss Milner y su marcha junto a su hija pequeña a un lugar recóndito de Es- 
cocia, Lord Elmwood decide nombrar al joven su heredero, proporcionándole la educación más esmerada. Cuando comienza la segunda parte de la novela nos encontramos con un muchacho inteligente, extremadamente atractivo y de modales exquisitos, que pronto se nos revela como el prototipo del «feminized man". Es una persona sensible, de profundos sentimientos, que respeta y quiere a Lord Elmwood por haberle protegido, recuerda con devoción y cariño a Miss Milner por lo que hizo por él y desde el primer momento que ve a Matilda sólo desea agradarla y someterse a sus deseos. Ya en su primer encuentro con Matilda muestra toda su ternura y delicadeza. Rushbrook está buscando un libro y un sirviente que sabe que Miss Woodley lo ha cogido para que lo lea Matilda se lo pide a la dama. Cuando el joven se entera de lo que ha hecho el criado, va inmediatamente a disculparse con Matilda, pues es consciente de que lo sucedido resulta insultante para la muchacha: “"If, Lady Matilda”, resumed he, "I have offended you, and must quit you without pardon, I am more unhappy than I should be with the loss of your father's protection - more forlorn, that when an orphan boy, your mother first took pity on me"". (238). La joven le trata con aspereza y él no sólo se deshace en un mar de lágrimas, rompiendo así con el esteorotipo del hombre fuerte, racional y capaz de controlar sus emociones, sino que adopta una actitud sumisa:

«... Professions, I find, have no weight, and only by this obedience to your orders can I give a proof of that respect which you inspire; - and let the agitation I now feel convince you, Lady Matilda, that, with all my seeming good fortune, I am not happier than yourself». - And so much was he agitated while he delivered this, it was with difficulty he came to the conclusion. - When he did, he bowed with reverence, as if he had left the presence of a deity, and went away. (239-40).

Este primer encuentro entre Rushbrook y Matilda no sólo sirve para que el lector se de cuenta de la sensibilidad del joven, sino para que se acreciente la admiración y el respeto que Rushbrook siente por Matilda. No entiende cómo es que Lord Elmwood puede tener apartado de él a este «jewel» que tan inmensamente feliz podría hacerle e incluso se plantea reconciliar al padre y la hija, aunque ello provoque su expulsión de la casa, puesto que Lord Elmwood no quiere ni ver a su hija ni que se pronuncie su nombre en su presencia: «Such was the youthful, warm, generous, grateful, but unthinking mind of Rusbrook». (241).

Las cosas se complican cuando Lord Elmwood le propone a Rushbrook que se case con una jovencita que él cree reúne todos los requisitos para ser una buena esposa y el muchacho no sabe qué contestar, puesto que está enamora- 
do de Matilda, cuyo nombre es anatema para su tío. Éste se impacienta con el joven y le da una semana para que se decida, lo que provoca que Rusbbrook caiga gravemente enfermo, dividido entre su incondicional devoción y obligación hacia Lord Elmwood, que le cuida con profundo cariño y preocupación durante su enfermedad, y su amor hacia Matilda, cuya situación de indefensión despierta su compasión.

Rushbrook vuelve a revelarse como un ser sensible que se preocupa por los que le rodean y no desea lastimar sus sentimientos, cuando se traslada a la casa que Lord Elmwood posee en el campo para terminar de recuperarse de su dolencia y se entera de que debido a su llegada Matilda ha tenido que recluirse en sus aposentos: «And yet, how could he support the idea that his visit had placed the daughter of his benefactor as a dependant stranger in that house, where in reality he was the dependant, and she the lawful heir». (258). Esta afirmación de Rushbrook resulta muy interesante, porque pone de manifiesto algo muy característico del «feminized man» y es su deseo de establecer no una relación jerárquica, sino igualitaria con su amada. De hecho, acorta su estancia en el campo y regresa a los pocos días a la ciudad «rather than by his stay placed in a subordinate state the object of his adoration» (258). Rushbrook no intenta, como otros personajes de la novela sentimental, acorralar y avasallar con sus apasionados sentimientos a su amada, sino que la trata con la mayor delicadeza y respeto. Ello se ve claramente cuando se encuentra por casualidad con Matilda durante un paseo: «In spite of the propensity he felt to pass so near to Lady Matilda, could he have known what conduct would have been deemed the most respectful, whatever painful denial it had cost him, that, he would have adopted». (265).

Cuando Lord Elmwood echa de su casa a Matilda, Rushbrook se plantea interceder por la joven, aunque ello genere su propia ruina, pero se da cuenta de que con su acción lo único que conseguirá es enfurecer más a su tío y, por tanto, perjudicar en mayor medida a la joven. Sin embargo, al cabo de unos días Rushbrook explota y le dice a Lord Elmwood lo que piensa: siente que ha usurpado el lugar y los sentimientos que le pertenecen a Matilda y no podrá casarse ni ser feliz mientras Matilda sea una huérfana, una exiliada, y él goce de todo aquello que realmente le pertenece a ella. Lord Elmwood se siente tremendamente dolido por las palabras de su sobrino, ya que cree que está siendo injusto con él al no entender la verdadera razón por la que ha actuado de la forma que lo ha hecho, y decide expulsarlo de la casa. La reacción en ese momento del muchacho es muy significativa, porque en lugar de enfrentarse a su tío lleno de soberbia y orgullo, como se esperaría en cualquier héroe que se precie, se siente apenado, culpable y merecedor del castigo impuesto porque es consciente de que ha herido terriblemente al hombre que tanto ha hecho por él 
There was a truth and a pathetic sound in the utterance of these words that struck Ruhsbrook to the heart - and he beheld himself as a barbarian, who had treated his benevolent and only friend, with an insufferable liberty; void of respect for those gnawing sorrows which had imbittered so many years of his life, and in open violation of his most strict commands. - He felt he deserved all he was going to suffer, and fell upon his knees, not so much to deprecate the doom he saw impending, as thus humbly to acknowledge it was his due. (291)

Yo no creo que Rushbrook se esté comportando aquí de forma completamente sumisa, como dice Ty, sino mostrando una gran fortaleza y coraje al admitir que se ha equivocado en el modo en que se ha dirigido a su tío y aceptando con valentía las consecuencias de su acto. El propio Lord Elmwood, que se define por su carácter rígido e inflexible, tras una conversación con Sandford decide perdonar al joven porque se da cuenta de la magnanimidad de sus sentimientos y de su tremenda generosidad al arriesgar todo lo que tiene por otra persona: «... I recollected there was a gratitude so extraordinary in the hazards he ran, that almost made him pardonable» (293). Incluso Sandford, un hombre que sólo acepta a aquellos que se adaptan a su ideal de conducta y que siempre ha denostado a Rushbrook por haber suplantado a Matilda como heredera, termina rindiéndose a su generosidad y le defiende delante de Matilda, diciendo que el muchacho sufre por todo lo que le está ocurriendo a ella y que Lord Elmwood no podría haber elegido a nadie mejor como su sucesor.

Cuando Rushbrook se entera de que el villano de Margrave está merodeando por los alrededores con la intención de seducir a Matilda, decide ir a la casa de su amada, aunque ello suponga poner nuevamente en peligro todo lo que tiene, pues sabe que su tío no quiere que tenga contacto con su hija. Rushbrook reconoce que su conducta no es la más apropiada, porque un caballero que se precie nunca debe visitar a una dama sin haber sido antes invitado, pero sabe que la situación es tan desesperada que no tiene otra opción. De hecho, cuando Miss Woodley le reprende severamente por haberse presentado de improviso, Rushbrook exclama: "...Do not imagine because you see me here, I approve of my conduct; but reduced to this necessity, pity the motives that have urged it». (309). Russhbrook le dice a Miss Woodley que si ella conoce otro modo de interesarse por la salud y el bienestar de Matilda que no sea el que él ha elegido, que se lo indique y él seguirá fielmente sus instrucciones. Nuevamente Rushbrook está demostrando aquí su tremenda sensibilidad y su deseo de actuar con la mayor delicadeza posible en todo lo concerniente a su amada para que ésta no se sienta agobiada u ofendida por sus atenciones hacia ella. Mrs. Woodley, consciente del buen corazón y ternura del joven deja que éste 
ve a Matilda y, de manera bastante evidente para el lector, que no para la muchacha, le manifiesta su incondicional amor y devoción: «She put out her hand - which he knelt to receive, but did not raise it to his lips - he held the boon too sacred - and only looking earnestly upon it, as it lay pale and wan in his, he breathed a sigh over it, and withdrew». (312).

Pero mientras que la pasión que Rushbrook siente por Matilda se acrecienta a medida que pasa el tiempo, Matilda sólo le ve como a un amigo a quien respeta:

She loved him as her friend, her cousin, her softer, but not as a lover. - The idea of love never once came to her thoughts; and she would sport with Rushbrook like the most harmless child, while he, all impassioned, could with difficulty resist telling her, what she made him suffer. (334)

De hecho, no sin cierta ironía, la narradora nos dice que durante el período que Matilda está prisionera de Margrave se acuerda de Miss Woodley y Sandford y sólo «Once she thought on Rushbrook too, and thought even he would be vext for her». (328), lo cual es un auténtico anticlímax y supone una clara subversión de la novela romántica, ya que lo que el lector espera de una heroína que se encuentra en una situación tan desesperada es que ansíe la llegada de su valeroso joven amado que la rescatara del villano que la tiene secuestrada. Matilda sólo despierta de su «letargo» y se da cuenta de que siente algo por Rushbrook cuando Lord Elmwood está a punto de echarlo nuevamente de la casa por confesar su amor por Matilda:

«Mr. Rushbrook is my relation», she cried in a pathetic voice, «my companion, my friend - before you loved me he was anxious for my happiness, and often visited me to propose some kindness. - I cannot see him turned out of your house without feeling for him, what he once felt for me». (336)

El hecho de que Matilda base su relación en el compañerismo y la amistad y no en el amor pasional es altamente relevante, porque a través de las palabras de Matilda Inchbald está defendiendo un tipo de matrimonio basado en principios más igualitarios, en el que la mujer juega un papel más activo y decisivo. Ros Ballaster lo ha explicado con gran claridad:

It is not until this traditionally gendered distribution of properties is reversed, establishing the male Rushbrook as the victim of sensibility and the female Matilda as the authoritarian presence, that this interpretative gridlock is overcome and a viable marital relationship posited. (213) 
Frente a Lord Elmwood que a través de su actitud autoritaria y represiva había convertido su casa en una auténtica prisión para los que la habitaban ${ }^{5}$, especialmente para aquellos que como Matilda y Rushbrook dependían de él, la joven pareja va a crear un hogar amoroso en el que no sólo prevalecerán valores como el respeto o la amistad, sino que además se invertirán las jerarquías existentes, otorgándosele una mayor autoridad a la heroína. Evidentemente, y eso Inchbald lo sabía muy bien, se trata simplemente de una quimera, de una fantasía de poder, pero ante una sociedad en la que los maridos tenían poder absoluto sobre sus mujeres y podían someterlas a las mayores vejaciones con la mayor impunidad, la imaginación era el único recurso que tenían las escritoras para subvertir una realidad que las esclavizaba económica, social y culturalmente.

\section{REFERENCIAS BIBLIOGRÁFICAS}

Armstrong, Nancy 1989: Desire and Domestic Fiction: A Political History of the Novel. New York: Oxford University Press.

BECKER, Susanne 1999: Gothic Forms of Feminine Fictions. Manchester: Manchester University Press.

Brownstein, Rachel M. 1994: Becoming a Heroine: Reading about Women in Novels. New York: Columbia University Press.

CASTLE, Terry 1986: Masquerade and Civilization: The Carnivalesque in Eighteenth-Century English Culture and Fiction. Stanford: Stanford University Press.

Figes, Eva 1988: Sex and Subterfuge. Women Writers to 1850. New York: Persea Books.

INCHBALD, Elizabeth 1998 (1791): A Simple Story. Ed. J.M.S. ToMPKINS, with an introduction by Jane Spencer. Oxford: Oxford University Press.

HoEveler, Diane Long 1998: Gothic Feminism. The Professionalization of Gender from Charlotte Smith to the Brontës. Pennsylvania: The Pennsylvania State University Press.

Kelly, Gary 1976: The English Jacobin Novel, 1780-1805. Oxford: Clarendon Press. MOERs, Ellen 1978: Literary Women. London: The Women's Press.

Moore, Catherine E. «Ladies... Taking the Pen in Hand». Mrs. Barbauld's Criticism of Eighteenth-Century Women Novelists. Fetter'd or Free? British Novelists, 1670-1815: 383-97.

Newton, Judith Lowder 1981: Women, Power, and Subversion: Social Strategies in British Fiction 1778-1860. Athens, Georgia: University of Georgia Press.

s Tr hace especial hincapié en este aspecto en su trabajo sobre A Simple Story. 
RoGERs, Katharine M. 1977: Inhibitions on Eighteenth-Century Women Novelists: Elizabeth INCHBALD and Charlotte SMITH. Eighteenth-Century Studies 11: 63-78.

Rogers, Katharine M. 1982: Feminism in Eighteenth-Century England. Urbana: University of Illinois Press.

Schofield, Mary Anne, \& Celina MACheski eds. 1986: Fetter'd or Free? British Women Novelists, 1670-1815. Athens, Ohio and London: Ohio University Press.

SCHOFIELD, Mary Anne 1990: Masking and Unmasking the Female Mind: Disguising Romances in Feminine Fiction, 1713-1799. Newark: University of Delaware Press.

Spacks, Patricia Meyer 1994: Desire and Truth. Functions of Plot in EighteenthCentury English Novels. Chicago \& London: University of Chicago Press.

Stone, Lawrence 1990: The Family, Sex and Marriage in England 1500-1800. Harmondsworth: Penguin.

ToDD, Janet 1989: The Sign of Angellica: Women, Writing and Fiction, 1660-1800. New York: Columbia University Press.

Tompkins, J. M. S. 1932: The Popular Novel in England 1770-1800. London: Constable \& Co.

Ty, Eleanor 1993: Unsex'd Revolutionaries: Five Women Novelists of the 1790s. Toronto: University of Toronto Press.

VAn LeEuwen, Frederike 1982: Female Gothic: The Discourse of the Other. Revista Canaria de Estudios Ingleses, 4: 33-44. 